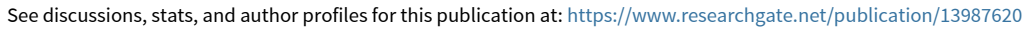

\title{
The development of a care-giving burden scale
}

Article in Age and Ageing · December 1994

Source: PubMed

\section{CITATIONS}

44

2 authors, including:

Peter C. Van der Ende

Hanzehogeschool Groningen

17 PUBLICATIONS 137 CITATIONS

SEE PROFILE

Some of the authors of this publication are also working on these related projects:

Evaluation Geriatric Assessment Unit View project

Support of fathers and mothers with mental illness and their personal and social recovery View project
READS

1,150 


\title{
The Development of a Care-giving Burden Scale
}

\author{
J. C. GERRITSEN, P. C. van DER ENDE
}

\begin{abstract}
Summary
A burden scale is described that is conceptually specific by concentrating on an assessment of different aspects of distress of care-giving burden and conceptually sensitive by distinguishing two dimensions: the relationship of the carer and the care-receiver and the limitations in the personal life of the carer.

To develop a burden scale an analysis was carried out on a database of an intervention study in which 89 informal caregivers of psychogeriatric patients were interviewed twice. A principal components analysis was carried out showing two main factors that could be interpreted as the two dimensions mentioned above. Reliability analysis showed a Cronbach's alpha of 0.84 for the total care-giving burden scale (13 items) and 0.77 for both subscales ( 7 and 6 items), confirmed in an independent sample. An analysis of the hierarchy of items (Mokken Scale Analysis) showed a strong and moderate hierarchy for the subscales and the total scale, respectively. Finally, an analysis of construct validity showed strong correlations of care-giving burden with depression of the carer and deviant behaviour of the patient.
\end{abstract}

\section{Introduction}

The concept of burden is important in care-giving research because the potentials of informal care are related to the risk of institutionalization $[1,2]$. Moreover, the concept focuses on the heavy impact of care-giving on informal carers. Depression is often found in populations of care-givers [3]. Empirical evidence with regard to burden can show the limitations of a policy aimed at reducing institutional care, or may indicate that these policy options cannot be realized without strong support for informal carers. Although there is consensus on the relevance of the concept, operationalizations vary widely among studies, both regarding the scope and the subjective or objective nature of the concept $[4,5]$. This variety and particularly the lack of specificity of the operationalization of burden impede an analysis of determinants and, by implication, the development of interventions.

The concept of burden presented in this contribution reflects only the subjective and affective dimensions, in contrast to objective and cognitive aspects [6]. Furthermore, the concept of burden is confined to so-called 'primary and secondary stressors' [7] of caregiving burden. An operationalization has been made of this concept based on a selection of items developed by Zarit et al. [8] and Vernooij-Dassen [9]. The measurement quality has been assessed using analyses of reliability and hierarchy of items and an analysis of construct validity.

\section{Burden: towards conceptual specificity}

The burden concept has been operationalized in many different ways. Poulshock and Deimling [10] used a narrow definition of the concept, i.e. emotional reactions to the care process; the approaches of many others include burden as a result of changes in the personal and social life of carers [8, 11-14]. A broader definition of the concept includes reactions to the impairment itself $[6,15-17]$ and negative aspects of social support $[18,19]$.

Moreover, conceptualizations and instruments differ with respect to their subjective and/or objective dimensions [20]. The subjective dimension of burden relates to (negative) emotional reactions or to cognitions, the objective dimension to 'disruptions to family/ household life that are potentially verifiable and observable' [21]. Often the two dimensions are combined [4].

The consequence of this conceptual diversity is that no unequivocal conclusions can be drawn with respect to the nature of the burden on care-givers and its relationship to the functioning of those receiving care and to other psychological characteristics of the carers themselves [4]. This implies that no sound basis emerges for interventions and care policy [6]. This underlines the need for a conceptualization of burden that is specific and sensitive to its different aspects.

To increase the specificity of the concept, only the subjective dimension should be operationalized, because distress is regarded as the core of agreement 
on what constitutes burden, i.e. 'worry, anxiety, frustration, depression, fatigue, poor health, guilt and resentment' [13]. The meaning of burden primarily relates to the subjective interpretation of informal carers and not to 'objective facts and circumstances' [10]. Moreover, the subjective dimension expresses the care-givers' personal experiences and their interpretation of the impact and meaning of care-giving. It can be assumed that the carers' assessment of burden should have more implications for their behaviour than an 'objective assessment' by others. The same objective situation can be experienced as difficult by one caregiver but not by another [6]. A direct assessment of distress provides an unequivocal picture in this respect.

Within the subjective dimension, the specificity of the concept can be improved further by concentrating on the affective aspects of care-giving burden, i.e. 'distress arising from dealing with the care-receivers' physical dependence and mental incapacity' $[6,7]$. This approach makes it possible to assess more clearly the impact on the carer of providing care because cognitions are excluded. It is not clear if and to what degree cognitions, although they are of a subjective nature, are stressful to individuals. For instance the item: 'You cannot leave your spouse alone, he/she needs you continuously', does not tap the way in which the supervision is stressful to the carer. When he or she does not mind remaining at home or when there are possibilities of having someone else to look after the patient occasionally, the situation will be less stressful. The item reformulated as: 'You feel yourself limited in your possibilities to go out, because your spouse needs you continuously' would be a more accurate indication of the impact of supervision on the carer. Therefore a combination of affect/distress and cognitions in the same instrument obscures the experience of problems by the informal care-giver and is vulnerable to contaminations of descriptions and evaluations [5].

Conceptual sensitivity can be enhanced by accounting for different dimensions within the care-giving burden. A unidimensional definition or representation of caregiving burden obscures its different components. Potentials for interventions will be limited, because specific aspects of burden cannot be distinguished. An instrument to measure care-giving burden should at least reflect distress in providing care and distress on the effects of care-giving over one's life [6]. This corresponds largely to the conceptualization of Pearlin et al. [7] of primary and secondary stressors related to care-giving. Primary stressors are conceived of as the emotions involved in care-giving itself, secondary stressors refer to the emotional perception of the consequences of care-giving for roles and activities outside care-giving. In this respect primary stressors are the direct consequence of care-giving, secondary stressors the indirect effects. Pearlin et al. [7] argued that this by no means implies that secondary stressors are of minor importance. The same conceptualization is present in the work of Whitlatch et al. [22]. They also found two dimensions: 'personal strain', reflecting primary stressors and 'role strain', which is close to secondary stressors. Conceptually, the distinction between primary and secondary aspects of care-giving burden can be used as a guide for instrument development that takes into account the main dimensions of burden. In the operationalizations the approach in this paper differs from those of Pearlin et al. [7] and Whitlatch et al. [22] because cognitions regarding care are excluded. In terms of distress, cognitions cannot be interpreted unequivocally. Moreover, the present approach of secondary aspects involves only 'feelings on impact' and not an assessment of 'intrapsychic strains' and 'the diminishment of self-concepts' [7]. In our view the effects on mental health or psychological changes should be operationalized and measured separately. Feelings about changes in health (impact), however, are secondary aspects of burden.

In this paper the development of an instrument to measure care-giving burden is described, based on earlier work $[8,9,23,24]$. A central question in this paper is how subjective care-giving burden as a narrower (sub)concept of burden can be operationalized while maintaining high or at least sufficient measurement qualities.

\section{Methods}

The items of a previously developed instrument [24] have been used as a starting point, because in this study the subjective aspect of care-giving burden is the central issue Vernooij-Dassen [9], however, chose a different way of measurement development which stressed 'competence' rather than 'burden'. In our study the same 27 items of Vernooij-Dassen are used to represent subjective aspects of care-giving burden, using the conceptual framework outlined in the Introduction.

The measurement quality of the instrument is assessed by analysing the reliability, construct validity and the hierarchical structure of the scales. Most often, only data on reliability have been reported. However, with scales comprising many items, high reliability is readily obtainable because of the socalled 'test-extension' effect [25]. 'Therefore, in addition to the analysis of reliability, the hierarchy of items within a scale should be analysed, because a distinct order of the items within a scale indicates a less arbitrary character of items and a comparable content (of items) for respondents when sum scores are equal.

The population of our study consisted of 89 carers of psychogeriatric patients, referred by general practitioners to a geriatric outpatient clinic of a psychogeriatric nursing home [26]. The main diagnoses of the patients were senile dementia of Alzheimer's type (SDAT) $(n=33)$, amnestic disorder $(n=13)$, organic psychosyndrome $(n=18)$ and multi-infarct dementia (MID) $(n=6)$. The carers were 60.1 years of age on average; $67(74 \%)$ of the carers were women. The relationships of carers and care-receivers were: 37 wives, 7 husbands, 24 daughters and 21 others. In our study two measurements were available, one (M1) conducted just before and one (M2) 3 months after the examinations in the outpatient clinic. Both measurements were used for the present analysis.

The analysis comprised five steps. The first step was to control the items for face validity. Secondly, factor analyses using varimax rotation were carried out. A third step was an analysis of reliability using Cronbach's alpha coefficient. 
The fourth step was to analyse the hierarchy of items within the total scale and the subscales. For this analysis Guttmanscaling is often used, which is based on a deterministic model of hierarchy. In this study, however, it was decided to use a procedure that accounts for errors due to chance. The Mokken Scaling Procedure (MSP) is based on a probabilistic model and produces a scalability statistic $\mathrm{H}$ [27-29]. When the $\mathrm{H}$ coefficient is high, the scales have a stronger hierarchical structure. This implies that equal sum scores for different respondents have a comparable content of items. Norms for the $\mathrm{H}$ coefficient are: a strong hierarchy if the coefficient is 0.50 or over, a moderate hierarchy if $\mathrm{H}$ ranges from 0.40 to 0.49 , a weak hierarchy with an $\mathrm{H}$ of 0.30 to 0.39 , no hierarchy if $\mathrm{H}$ is lower than 0.30 [30]. The MSP programme also gives $\mathrm{H}(\mathrm{i})$ coefficients for individual items, i.e. their contribution to the hierarchy of the scale. In addition, the MSP provides a direct estimation of reliability by the rho coefficient. The rho can be conceived as an improvement on the common Cronbach's alpha coefficient, because the latter represents a lower boundary of reliability.

The fifth step consists of the evaluation of the construct validity of the instruments developed. First, the relationships between the concept of care-giving burden and its two subdimensions, relationship and personal consequences, are explored. It is assumed that both are related but not very strongly. Moreover, the relationships of the total and the subscales with health indicators of the carers and the carereceivers have been evaluated.

Positive correlations were expected between care-giving burden on the one hand and depression of the carer, deviant behaviour and memory and orientation of the care-receiver on the other. In particular, a strong impact of deviant behaviour on care-giving burden and its subscales was expected. Lower correlations were expected for care-giving burden and ADL of the care-receiver, because ADL care is assumed to be not a very great source of tension as there is less uncertainty.

Depression of the carers was assessed with the selfreporting depression scale of Zung [31]. A sufficient reliability was found $(\alpha=0.74)$. Characteristics related to the needs of the care-receiver were assessed in the interview with the informal carer. ADL of the care-receiver has been assessed with a polychotomous scale developed by Kempen and Suurmeijer [32] (Reliability $\alpha=0.87$ ). Deviant behaviour and memory and orientation of the care-receiver have been assessed with four-item subscales of a behavioural problems checklist $[33,34]$. Deviant behaviour consisted of four items: 'suspicion', 'anger', 'reluctance' and 'difficult to get on with'. Memory and orientation included a lack of orientation in time, place and person and memory problems. Answer categories were 'never', 'seldom', 'sometimes', 'often/ always'. Reliabilities of the deviant behaviour scale and the memory and orientation scale were: $\alpha=0.81$ and $\alpha=0.76$ [26].

\section{Results}

Selection of items: Vernooij-Dassen started her study by selecting 55 items on subjective care-giving burden from the Zarit Burden interview [8, 23], and other items, partly based on the social breakdown model of Bengtson and Kuypers [35]. These items were judged by a panel comprising academics, care-providers and students $(n=39)$. After factor analysis an instrument called 'Sense of competence' was constructed comprising 27 items and having good reliability $(\alpha=0.88)[9,24]$.
These 27 items were the starting point for a screening on face validity of care-giving burden and (direct assessment of) distress carried out by the researchers with the aid of colleagues. All 27 items relate to caregiving burden. Seven of the 27 items were cognitions and did not assess emotional reactions explicitly and directly; they were therefore deleted. Three items referred to evaluations, for instance 'Because of my involvement with my spouse, I don't have time for myself'. Three other items reflected opinions on the care-receiver, for instance ' $\mathrm{My}$ spouse wants to manipulate me', and one item related to a desired future situation: 'I wish my spouse and I had a better relationship'.

Results of the factor analysis: A principal components analysis was performed in both measurements on the remaining 20 items. The results of the factor analysis are summarized in Table I. The numbers of the items correspond to the numbers in the Appendix. In both measurements there were six factors explaining $60.4 \%$ (M1) and $62.5 \%$ (M2) of variance, respectively. Only the first two factors, however, could be interpreted. A strong first factor in the first measurement and a weaker factor in the second measurement-'Personal Consequences'-is the subjective impact of care-giving on the lives of the carers. A second factor in the first measurement and a strong first factor in the second measurement is 'Relationship', i.e. a negative evaluation of the care relationship. These two factors explained $34.4 \%$ (M1) and $37.6 \%(\mathrm{M} 2)$ of variance. It can be concluded that there are two factors in both measurements that reflect the conceptual distinction of 'primary' and 'secondary' aspects of care-giving burden.

The differences of the factor structure, in particular the varying importance of factors, need attention. In general, the fact that a number of items have been selected as a first factor means that the correlations between items are higher than the correlations of items on the second factor (and other factors). Probably the increasing coherence of items on 'Relationship' and decreasing coherence of items on 'Personal Consequences' can be explained by the intervention of the outpatient clinic. After the intervention more attention may have been given to the impact of the treatment and its effects on the interaction of patients and carers. Moreover, on some aspects of personal consequences a change was established by the intervention of the outpatient clinic [26]. The intervention included special attention to carers by a psychologist.

Based on the factor analyses, six items have been selected for analysis of reliability with respect to personal consequences $(1,2,4,6,7,11)$ and eight items for relationship $(3,5,8,9,10,12,13,15)$. Items were selected when they loaded high enough on the same factor in both measurements in the first analysis and when the content reflected the conceptual distinction between primary and secondary aspects. Two exceptions have been made because of the content of the items 


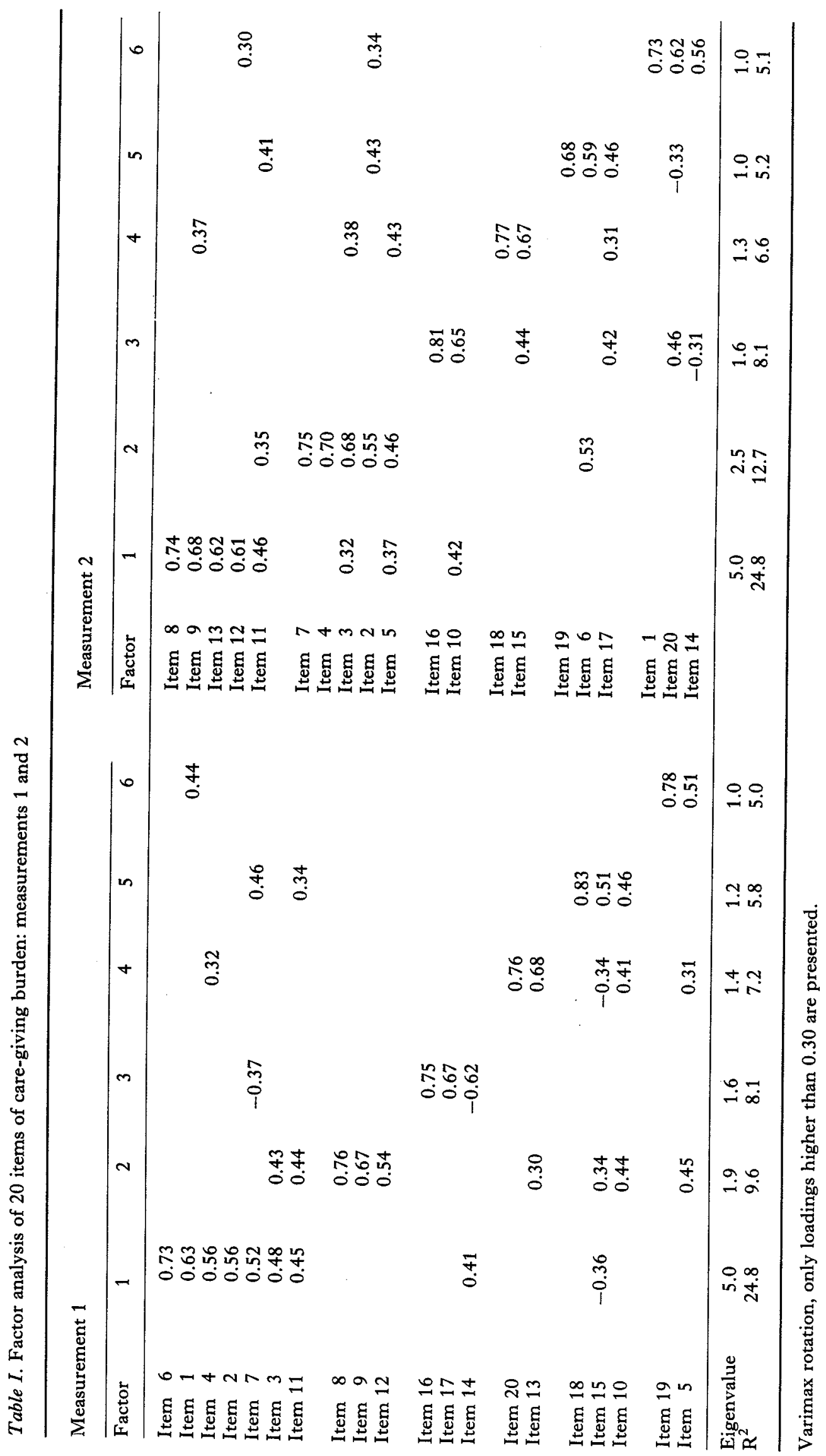


item 1 has been allocated to personal consequences (' $I$ never feel free from the care for my spouse') and item 15 to relationship ('I feel useful in my interactions with my spouse'). Both items loaded on the respective factors in one of both measurements. When an item loaded on two factors in the two measurements (items 3 and 11) decisions were made by looking at the content of the items (provided the factor loadings were satisfactory). Item 3 relates to 'strain' in the relationship with the elderly person and has been allocated to 'Relationship'. Item 11 refers to 'health consequences' for the informal carer and has been allocated to 'Personal Consequences'. Item 14 was dropped because of an unexpected negative loading on factor 1 indicating potential technical or validity problems. The remaining five items did not load on both factors. Moreover, there was no other reason (for instance skewed distribution, but adequate content) to justify inclusion in either factor. Therefore, these five items were dropped.

Results of the analysis of reliability and the hierarchy of items: An analysis of reliability was carried out in both measurements using Cronbach's alpha and the rho coefficient. Hierarchy was assessed using the MSP programme. The results, i.e. the means and $H$ coefficients of the individual items are presented in Table II. The hierarchy of items is almost equal in the two measurements. Therefore, only MSP analysis of the first measurement will be presented below. Deviations will be accounted for.
The total 'Care-giving Burden Scale' consists of 13 items and has an adequate reliability in both measurements as assessed with Cronbach's alpha and the rhostatistic. It was decided to drop item 15 to increase reliability in both measurements. The total scale has a moderate hierarchy ( $\mathrm{H}$ coefficient). The order of items is almost the same in both measurements, except for items 3 and 6 which were a little more difficult in the first measurement and item 8 which was somewhat more difficult in the second measurement.

The first subscale 'Relationship' has sufficient reliability. It consists of seven items and has a moderate to strong hierarchy of items. The order of items is the same in both measurements, except for items 3 and 8 .

The second subscale 'Personal Consequences' has a good reliability. Here the rho (direct indicator) and alpha statistic (lower boundary) differed considerably. However, by the standards of the alpha, reliability is still adequate. The hierarchy of items in this subscale is strong in both measurements. The order of items is consistent in both measurements except for item 6 .

Because the reliabilities were calculated on the (same) sample that was used for scale construction, it was decided to control the reliabilities on an independent sample, containing the same items as those that were included in the scales. A new analysis has been carried out on a small sample of carers of diagnosed Alzheimer patients $(n=42,60.5$ years of age on average, $62 \%$ are women, $34 \%$ are partners of the patients) [36]. Because

Table II. Results of the reliability and MSP analysis of the Care-giving Burden Scale and subscales: Relationship and Personal Consequences, two measurements $[M 1(n=89)$ and M2 $(n=89)]$

\begin{tabular}{|c|c|c|c|c|c|c|c|c|}
\hline \multicolumn{3}{|c|}{ Care-giving Burden } & \multicolumn{3}{|c|}{ Relationship } & \multicolumn{3}{|c|}{ Personal Consequences } \\
\hline $\begin{array}{l}\text { Item } \\
\text { no. }\end{array}$ & Mean & $\mathrm{H}(\mathrm{i})$ & $\begin{array}{l}\text { Item } \\
\text { no. }\end{array}$ & Mean & $\mathbf{H}(\mathrm{i})$ & $\begin{array}{l}\text { Item } \\
\text { no. }\end{array}$ & Mean & $H(i)$ \\
\hline 1 & 0.74 & 0.61 & 3 & 0.57 & 0.59 & 1 & 0.74 & 0.71 \\
\hline 2 & 0.62 & 0.60 & 5 & 0.40 & 0.43 & 2 & 0.62 & 0.62 \\
\hline 3 & 0.57 & 0.54 & 8 & 0.36 & 0.54 & 4 & 0.53 & 0.51 \\
\hline 4 & 0.53 & 0.51 & 9 & 0.35 & 0.51 & 6 & 0.40 & 0.44 \\
\hline 5 & 0.40 & 0.39 & 10 & 0.26 & 0.46 & 7 & 0.37 & 0.48 \\
\hline 6 & 0.40 & 0.31 & 12 & 0.20 & 0.34 & 11 & 0.25 & 0.63 \\
\hline 7 & 0.37 & 0.37 & 13 & 0.20 & 0.54 & & & \\
\hline 8 & 0.36 & 0.47 & & & & & & \\
\hline 9 & 0.35 & 0.42 & & & & & & \\
\hline 10 & 0.26 & 0.46 & & & & & & \\
\hline 11 & 0.25 & 0.53 & & & & & & \\
\hline 12 & 0.20 & 0.35 & & & & & & \\
\hline \multirow[t]{2}{*}{13} & 0.20 & 0.54 & & & & & & \\
\hline & M1 & M2 & & M1 & M2 & & M1 & M2 \\
\hline $\mathrm{H}$ & 0.46 & 0.48 & & 0.49 & 0.51 & & 0.55 & 0.60 \\
\hline Rho & 0.86 & 0.87 & & 0.78 & 0.81 & & 0.80 & 0.83 \\
\hline Alpha & 0.84 & 0.83 & & 0.77 & 0.77 & & 0.74 & 0.77 \\
\hline Mean & 5.58 & 5.04 & & 2.48 & 2.36 & & 3.07 & 2.78 \\
\hline $\mathrm{SD}$ & 3.54 & 3.45 & & 2.11 & 2.06 & & 1.87 & 1.80 \\
\hline
\end{tabular}


of the chance that deviant patterns have a larger impact in smaller sample sizes, the test of reliability is more critical than in larger sample sizes. In the analysis sufficient reliabilities were found (Care-giving Burden Scale: $\alpha=0.84$; relationship $\alpha=0.77$; personal consequences $\alpha=0.75$ ).

Construct validity: In Table III the results of the analysis of construct validity are presented. It was decided to conduct analyses for both measurements, but to report only the results of the first measurement since the results proved comparable. The data on the mental health and functional ability of the carereceivers have been collected in interviews with the carers. Therefore these data represent perceptions of the carer and not an 'objective' assessment.

The coherence of the dimensions of the concept of care-giving burden can be evaluated. Both dimensions have high positive correlations with the total scale $(0.90$ and 0.87$)$. The correlation between the two subdimensions is substantial but not very high $(0.58)$, confirming the distinct character of the two dimensions.

There are positive correlations between care-giving burden and depression, deviant behaviour and memory and orientation. These correlations were particularly high with respect to deviant behaviour and depression. Technically a correlation does not indicate a direction of the relationship. Depression can be either conceived of as a (strong) predisposing factor to or a consequence of burden. Correlations with ADL were not significant for Care-giving Burden. The correspondence of burden and depression of carers has also been found by others $[10,18,37]$. A relationship with deviant behaviour of the care-receiver has been found by Pearson et al. [38], while Vitaliano et al. [37] and Kinney and Stephens [18] found a correspondence between cognitive functioning (memory and orientation) and burden.

Deviant behaviour was most strongly related to Relationship and somewhat less strongly to Personal consequences. The difference between both correlations is not significant. Depression shows a strong relation to both Personal Consequences and Relationship.

The correlation of memory and orientation with care-giving burden is in accordance with expectations. Memory and orientation problems make continuous supervision necessary. ADL of the care-receiver relate only (weakly) to Personal Consequences. The lack of strong correlations with Relationship confirms our expectations.

\section{Conclusions}

In this study a Care-giving Burden Scale has been developed, taking into account three conceptual conditions: (1) the instrument reflects a direct assessment of distress, (2) the instrument concentrates on care-giving burden and not on burden by impairment, and (3) the instrument is multidimensional and distinguishes primary (care-giving itself) and secondary aspects (consequences for other activities and roles) of care-giving burden.

The Care-giving Burden Scale consists of 13 items and comprises two subscales: 'Relationship' (seven items) and 'Personal Consequences' (six items). The psychometric qualities of the Care-giving Burden Scale and its subscales are satisfactory. The reliability of the total scale and its subscales is relatively high. Therefore, these subscales can be used as separate measures. In this way a more detailed analysis of care-giving burden is possible. Within all scales a moderate to strong hierarchy of items was found. This implies that there is a strong probability that the individual scores on items are the same with equal sum scores. This adds to the measurement quality of the scales because the possibilities for comparison of scores are increased.

An analysis of the construct validity of the caregiving burden scale showed high positive correlations with deviant behaviour of care-receivers and depression of carers and somewhat less high positive correlations with memory and orientation. There was no significant correlation with ADL of the care-receiver.

\section{Discussion}

We propose a concept of burden that is limited to affective components and to primary and secondary

Table III. Construct validity of Care-giving Burden: total scale and subscales, Pearson correlations

\begin{tabular}{|c|c|c|c|c|c|c|c|}
\hline & 1 & 2 & 3 & 4 & 5 & 6 & 7 \\
\hline $\begin{array}{l}\text { 1. Care-giving Burden } \\
\text { 2. Relationship }\end{array}$ & $\overline{0.90}$ & - & & & & & \\
\hline 3. Personal Consequences & 0.87 & 0.58 & - & & & & \\
\hline 4. Depression & 0.53 & 0.43 & 0.52 & - & & & \\
\hline \multicolumn{8}{|l|}{$\begin{array}{l}\text { Care receiver } \\
\text { 5. ADL }\end{array}$} \\
\hline 5. ADL & 0.18 & 0.08 & 0.24 & 0.21 & - & & \\
\hline 6. Deviant behaviour & 0.53 & 0.53 & 0.40 & 0.27 & 0.01 & - & \\
\hline 7. Memory/orientation & 0.31 & 0.19 & 0.36 & 0.35 & 0.36 & 0.25 & - \\
\hline
\end{tabular}

All correlations $r>0.24$; significant $\mathrm{p}<0.00$.

Correlations: ADL with Personal Consequences and Depression; Memory and orientation with Relationship, $p<0.05$. 
stressors. Its operationalization has resulted in a new preliminary instrument to assess care-giving burden. This construct should be further analysed in larger and independent samples before it can be accepted as a standard instrument.

For the present, the Care-giving Burden Scale provides a specific viewpoint for the evaluation of stressors experienced by the care-givers directly related to the care process. An improvement seems to be that this assessment is not contaminated by objective evaluations of health, income and social support. When the latter variables are considered as essential in the light of research questions they should be operationalized separately. With the specific operationalization, determinants of care-giver burden can be identified and interventions can be developed that are directly attuned to these determinants. Results of the construct-validity analysis suggest that deviant behaviour of the elderly patient might be such a determinant. Interventions then might include for instance the development of noncorrective emphatic interaction styles for informal carers. The Care-giving Burden Scale in its present form therefore has potentially scientific relevance (specificity of the analysis of burden) as well as practical relevance (development of interventions). Further work on measurement quality, however, is a first priority.

In order to refine the provisional instrument, attention should be paid to at least two issues. First the theoretical domain of the (sub)scales should be assessed. Does the present selection of items provide a complete assessment of the primary and secondary stressors, respectively? This issue is particularly important because the operationalization of the secondary stressors (Personal Consequences) is culture-dependent and reflects in its present form a culture in which health, social participation and privacy are highly valued. In contrast, competing demands like work and family might be under-represented (one combined item). Although a uniform construct has the advantages of (cross-cultural) comparison, it may neglect issues that are relevant to other cultures. One strategy is to formulate additional items so that both comparison and a specific cultural assessment are possible. The same solution might apply to another issue in the measurement of burden, i.e. the specific backgrounds of carers with respect to sex, generation and relationship. Various studies have revealed that these carer backgrounds are related to the nature and level of burden [39-41]. These studies show that burden is related to loneliness and depression for wives, whereas burden for children is related to conflicting demands. Moreover, the level of burden for daughters seems higher than for wives due to additional obligations to their own families and social life [41]. Men are under-represented as carers compared with women. Available data, however, indicate that burden is higher for women than for men. An explanation might be that burden for men is associated with instrumental and practical tasks, while they are able to maintain emotional distance from the care- receiver, whereas women become more emotionally involved [40]. Because the Care-giving Burden Scale has two domains, the different nature of burden for the respective subgroups of carers will be reflected by different scores on Relationship and Personal Consequences. In a similar way as for culture it might be hypothesized that particularly with respect to Personal Consequences there are aspects of burden that need a more elaborate operationalization for a particular subgroup of carers. The particular problem of 'women in the middle' [42]-daughters of carereceivers who also have their own families to care for-warrants additional items or a subscale to capture this specific aspect of burden. Furthermore, for the spouse who is a co-resident the stress stemming from continuous supervision, changes in the marital relationship and isolation needs more attention in the operationalization. We propose to use a common standard that applies to all carers in order to make valid comparisons and in addition to develop items or subscales to capture subgroup-specific aspects of burden. In summary, in further work on the Caregiving Burden Scale the proposed instrument should be tested on independent populations, and new additional scales or items using the conceptual approach of affect/ distress are to be developed to assess more culture- and subgroup-specific components of care-giving burden.

\section{Acknowledgements}

The authors wish to thank M. J. F. J. Vernooij-Dassen, B. P. te Velde and H. E. P. Bosveld for their contributions and comments. This study was made possible by a grant from the Ministry of Welfare, Health and Culture, Rijswijk, The Netherlands.

\section{References}

1. Colerick EJ, George LK. Predictors of institutionalization among caregivers of Alzheimer patients. $\mathcal{F}$ Am Geriatr Soc 1986;34:493-8.

2. George LK, Maddox G. Aspects of institutional care. In: Ory MG, Bond K, eds. Aging and health care. London/ New York: Routledge, 1989.

3. Cohen D, Eisdorfer C. Depression in family members caring for a relative with Alzheimer's disease. $\mathcal{I} \mathrm{Am}$ Geriatr Soc 1988;36:885-9.

4. Vitaliano PP, Young HM, Russo J. Burden: a review of measures used among caregivers on individuals with dementia. Gerontologist 1991;31:67-75.

5. Stephens MAP, Kinney JM. Caregiver stress instruments: assessment of content and measurement quality. Gerontol Rev 1989;2(1):40-54.

6. Braithwaite V. Caregiving burden: making the concept scientifically useful and policy relevant. Res Aging 1992;14:3-27.

7. Pearlin LI, Mullan JT, Semple SJ, Skaff MM. Caregiving and the stress process: an overview of concepts and their measures. Gerontologist 1990;30:583-94.

8. Zarit SH, Reever KH, Bach-Peterson J. Relatives of the impaired elderly: correlates of feelings of burden. Gerontologist 1980;20:649-55.

9. Vernooij-Dassen MJFJ. Dementie en thuiszorg. Lisse/ Amsterdam: Swets \& Zeitlinger, 1993. 
10. Poulshock SW, Deimling T. Families caring for elders in residence: issues in the measurement of burden. 7 Gerontol 1984;39:230-9.

11. Robinson B. Validation of a caregiver strain index. $\mathcal{J}$ Gerontol 1983;38:344-8.

12. Montgomery RJV, Gonyea JG, Hooyman NR. Caregiving and the experience of subjective and objective burden. Fam Relat 1982;34:19-26.

13. Lawton MP, Kleban MH, Moss M, Rovine M, Glicksman A. Measuring caregiver appraisal. F Gerontol 1989;44: 61-7.

14. Novak M, Guest C. Application of a multidimensional caregiver burden inventory. Gerontologist 1989;29:798803.

15. Argyle N, Jestice S, Brooke CPB. Psychogeriatric patients: their supporters' problems. Age Ageing 1985; 14:355-60

16. Gilleard CJ, Gilleard E, Gledhill K, Whittick J. Caring for the elderly mentally infirm at home: a survey of their supporters. I Epidemiol Community Health 1984;38:31925.

17. Greene JG, Smith R, Gardiner M, Timbury GC. measuring behavioural disturbance of elderly demented patients in the community and its effects on relatives: a factor analytic study. Age Ageing 1982;11:121-6.

18. Kinney J, Stephens MAP. Caregiving hassles scale: assessing the daily hassles of caring for a family member with dementia. Gerontologist 1989;29:328-32.

19. Janssen T. Thuiszorg ' $n$ hele zorg, opvattingen en ervaringen van centrale verzorgers van hulpbehoevende oudere mensen, Nijmegen, 1988.

20. Thompson $\mathbf{H}$, Doll $W$. The burden of families coping with the mentally ill: an invisible crisis. Fam Relat 1982;31:379-88.

21. Platt $\mathrm{S}$. Measuring the burden of psychiatric illness on the family: an evaluation of some rating scales. Psychol Med 1985;15:383-93.

22. Whitlatch CJ, Zarit SH, von Eye A. Efficacy of interventions with caregivers: a reanalysis. Gerontologist 1991;31:9-14.

23. Zarit JM, Zarit SH. Measurement of burden and social support. Paper presented at the annual scientific meeting of the Gerontological Society of America. San Diego, 1982.

24. Vernooij-Dassen MJFJ, Persoon J. Het thuismilieu van dementerende ouderen. Instituut voor Sociale Geneeskunde/Nijmeegs Huisartsen Instituut, Katholieke Universiteit Nijmegan, 1990.

25. Groot AD de. Methodologie. Den Haag: Mouton, 1961.

26. Gerritsen JC, Van der Ende PC, Wolffensperger EW, Boom RCh. Evaluatie van een polikliniek psychogeriatrie. NCG/GCG, Groningen, 1992.

27. Debets P, Brouwer E. MSP: a program for Mokken Scale Analysis for polychotomous items. Amsterdam: Technisch Centrum. University of Amsterdam, 1987.

28. Mokken RJ. $A$ theory and procedure of scale analysis. The Hague: Mouton, 1971.

29. Molenaar IW. Mokken scaling revisited. Kwantitatieve Methoden 1982;3:145-64.
30. Jong A de, Molenaar IW. An application of Mokken's model for stochastic cumulative scaling in psychiatric research. F Psychiatr Res 1987;21:137-49.

31. Zung WWK. A self-rating depression scale. Arch Gen Psychiatry 1965;12:63-70.

32. Kempen GIJM, Suurmeijer ThPBM. The development of a hierarchical polychotomous ADL-IADL scale for noninstitutionalized elders. Gerontologist 1990;30:497502.

33. Wolffensperger EW, Van den Heuvel WJA, Gerritsen JC. Versterking van de sociale psychogeriatrie. Vakgroep Medische Sociologie, Groningen, 1987.

34. Gerritsen JC, Wolffensperger EW, Van den Heuvel WJA. Rural-urban differences in the utilization of care by the elderly. $f$ Cross-Cultural Gerontol 1990;5:13147.

35. Bengtson VL, Kuypers J. Change, competence, crises and intervention: a systems model of aging and family relations, Paper at the International Pre-Congress Workshop on Life-span and Change in Gerontological Perspective. Nijmegen. Vakgroep Sociale Gerontologie, 1981.

36. Hadderingh E, Kootte M, te Velde BP, Kempen GIJM, Van den Bremen WM. De zorg aan thuiswonende dementerenden. Groningen: Dementie Onderzoek in Groningen, 1991.

37. Vitaliano PP, Russo J, Young HM, Becker J, Maiuro RD. The screen for caregiver burden. Gerontologist 1991;31: $76-83$

38. Pearson T, Verma S, Nellett C. Elderly psychiatric patient status and caregiver perceptions as predictors of caregiver burden. Gerontologist 1988;28:79-83.

39. Morris RG, Morris LW, Britton PG. Factors affecting the emotional wellbeing of the caregivers of dementia sufferers. Br $\mathcal{F}$ Psychiatry 1988;153:147-56.

40. Harper S, Lund DA. Wives, husbands, and daughters caring for institutionalized and noninstitutionalized dementia patients: toward a model of caregiver burden. Int F Aging Hum Dev 1990;30:241-62.

41. Jones DA, Peters TJ. Caring for elderly dependants: effects on the carers' quality of life. Age Ageing 1992;21: 421-8.

42. Brody EM. 'Women in the middle' and family help and older people. Gerontologist 1981;21:471-81.

Authors' addresses

J. C. Gerritsen

Northern Centre for Health Care Research (NCH),

p/a Academisch Ziekenhuis Groningen,

Gebouw 37 1le Verdieping, Postbus 30.001,

9700 RB Groningen, The Netherlands

P. C. van der Ende

Groningen Centre for Gerontology (GCG),

Hanzehogeschool, Rijkshogeschool

van Groningen, The Netherlands

Received in revised form 18 April 1994 


\section{Appendix. Care-giving Burden Scale (CBS)}

The items are presented in order of difficulty, first the so-called easy items, then the more difficult ones (see Table I).

*1. I never feel free from the care for my spouse (V)

*2. The responsibility for my family, my other family members, my job etc. and on top of it the responsibility for my spouse is a heavy burden on me $(B / V)$

$\dagger 3$. I feel strained in my interactions with my spouse $(Z)$

*4. I feel nervous or depressed about my interactions with my spouse $(Z)$

†5. I feel pleased about my interactions with my spouse ( $Z$, to be recoded in opposite direction)

*6. I feel that the present situation with my spouse doesn't allow me as much privacy as I'd like (Z)

*7. I feel that my social life has suffered because of my involvement with my spouse $(Z)$

†8. I feel that my spouse makes requests which I perceive to be over and above what he/she needs $(Z)$

†9. I feel angry about my interactions with my spouse $(Z)$

†10. I feel that my spouse doesn't appreciate what I do for him/her as much as I would like (Z)

*11. I feel that my health has suffered because of my involvement with my spouse $(Z)$

†12. I feel embarrassed over my spouse's behaviour $(Z)$

+13 . I feel resentful about my interactions with my spouse $(Z)$

Subscales: *Personal Consequences; † Relationship

Response categories: 1 disagree very much; $2=$ disagree; $3=$ agree on the one hand, disagree on the other; $4=$ agree; $5=$ agree very much (recoded to dichotomies $(1,2=0)(3,4,5=1)$.

$\mathrm{Z}=$ Zarit, $\mathrm{V}=$ Vernooij-Dassen, $\mathrm{B} / \mathrm{V}=$ Vernooij-Dassen based on the model of Bengtson and Kuypers.

Items loading on one of the two main factors that have been deleted for technical reasons (14) and reliability (15):

14. I feel that my spouse seems to expect me to take care of him/her as if I were the only one he/she could depend on (Z)

15. I feel useful in my interactions with my spouse ( $Z$, to be recoded in opposite direction).

The other five items that were included in factor analysis but loaded neither factor:

16. I feel that I'm not capable to care for my spouse (B/V)

17. I feel that I don't do as much for my spouse as I should or must do (B/V)

18. I feel that in the past I haven't done as much for my spouse as I should or must have done $(Z)$

19. I feel guilty about the interactions with my spouse (Z)

20. I feel that whatever I do my spouse does not benefit $(B / V)$. 